\title{
The Role of Cardiolipin Remodeling in Mitochondrial Function and Human Diseases
}

\author{
Yan Yan $^{1} \&$ Bin Kang ${ }^{2}$ \\ ${ }^{1}$ Cell and Developmental Biology Program, the Pennsylvania State University, University Park, Pennsylvania, \\ USA \\ ${ }^{2}$ Department of Biology, University of Pennsylvania, Pennsylvania, USA \\ Correspondence: Yan Yan, Cell and Developmental Biology Program, the Pennsylvania State University, \\ University Park, Pennsylvania 16802, USA. Tel: 1-717-343-2307. E-mail: yanyanms@gmail.com
}

Received: April 29, $2011 \quad$ Accepted: May 18, $2011 \quad$ Published: November 6, 2012

doi:10.5539/jmbr.v2n1p1 URL: http://dx.doi.org/10.5539/jmbr.v2n1p1

This work is supported by Penn State University

\begin{abstract}
Cardiolipin (CL), one of bisphosphatidyl glycerol lipid, is found to contain high level of C18:2 acyl chains and exist exclusively in inner membranes of mitochondria. Cardiolipin remodeling is the process for CL to attain its specific acyl chains. With the purpose of introducing this interesting field to readers, in this review, we elucidate the role of cardiolipin in normal functions of mitochondria by analyzing the physical and chemical characters of cardiolipin; we introduce the current models of cardiolipin remodeling by analyzing the enzymes involved in this process; we reviewed the latest progress in the clinical implications of cardiolipin remodeling.
\end{abstract}

Keywords: Cardiolipin, Cardiolipin remodeling, Mitochondria, human diseases

Cardiolipin (CL) is one type of bisphosphatidyl glycerol lipid, which is in the name of the heart tissue since it was firstly isolated from bovine heart in 1942. CL exists throughout the eukaryotes - animals, plants and fungi. Cardiolipin contains high level of C18:2 acyl chains, indicating that de novo synthesized CL require an acyl-CoA remodeling process. Due to its unique highly unsaturated fatty acyl chain, CL plays important roles in functions of mitochondria including ATP biosynthesis and apoptosis. Dysfunction of CL remodeling caused by loss of the high level of unsaturated acyl chains are found in many pathological situations such as aging, heart failure and Barth syndrome.

\section{CL Dimmeric Structure and Its Physical and Chemical Characters}

CL belongs to a subfamily of phospholipids whose backbones and head groups are formed from multiple units of phosphoric and glycerol moieties, so they are called polyglycerophospholipids. Polyglycerophospholipids members include cardiolipin, phosphatidic acid, phosphatidylglycerol, and their lysocompounds, as well as bis (monoacylglycero) phosphate, acylphosphatidylglycerol, and phosphatidylglycerophosphate. The existence of many hydroxyl groups in this phospholipids subfamily makes it possible to form multiple positional and steric isomers (Schlame, 2008).

Containing a glycerol backbone linking two phosphatidyl moieties at the 1,3 positions, CL forms unique dimeric structure (LeCocq et al., 1964). Each phosphatidyl moiety contains two distinct alkyl groups, so CL potentially can have many species in cells. However, the composition of acyl groups in CL exhibits a notable pattern with a high percentage of $\mathrm{C} 18$ chains. The main specie of acyl chain in mammalian tissues is linoleic acid (Hoch, 1992; Schlame et al., 2000). In bovine heart, linoleic acid comprises about $80 \%$ of all acyl chains (Keenan et al., 1970). The abundance of CL containing four C18 and two unsaturated linoleic acid ester residues in animal cells indicates these unsaturated fatty acyl residues maybe are necessary for the function of CL in inner membrane of mitochondria (Schlame et al., 2000; Pangborn, 1942).

Four acyl chains and two phosphorus groups of CL do not show a symmetrical structure, nor does the CL exhibit all four possible configurations: R/R, R/S, S/S and S/R. The natural CL exists only in R/R mirror configuration. The two phosphorus atoms in CL are stereo chemically nonequivalent even in the presence of four same acyl 
chains (LeCocq et al., 1964; Powell et al., 1974). The acidity of two phosphorus groups are also different, $\mathrm{pK}_{1}$ $=3$ and $\mathrm{pK}_{2}>7.5$; CL is thus negative charged in the physiological environment. At different conditions such as non-neutral $\mathrm{pH}$, high or low ion strength or divalent cations, $\mathrm{CL}$ can form either lamellar or inverted hexagonal structures (Schlame et al., 2000; Ortiz et al., 1999). Nuclear magnetic resonance (NMR) and X-ray crystallographic data indicated that CL exists in a bilayer conformation; moreover, the two outer glycerol groups have identical orientation perpendicular to the membrane plane (Ortiz et al., 1999). The physical characters of CL make it possible to interact with many different proteins, lipids to form different membrane domains to facilitate different biological functions.

\section{Physiological Roles of CL Remodeling}

$\mathrm{CL}$ was exclusively distributed in the inner membrane of mitochondria of all eukaryotes. It accounts for about $20 \%$ of the total lipid in the mitochondria inner membrane. Studies suggested that CL of mitochondria is vital to the oxidative phosphorylation, apoptosis and other functions of mitochondria. Change in CL contents of mitochondria has been reported in many disorders including Barth Syndrome, Parkinson disease, heart failure, and male sterility.

$\mathrm{CL}$ is required for maintaining of proper mitochondrial membrane potential. The reduced membrane potential caused by the absence of CL leads to defects in protein import and other mitochondrial functions (Jiang et al., 2000). CL has a high $\mathrm{pK}_{2}$ (above 7) so its head group was found to be a proton trap in the membrane where protons are pumped. The head group domain supplies protons for ATP synthase with minimal change in the bulk phase $\mathrm{pH}$ (Haines et al., 2002).

$\mathrm{CL}$ is required for the function of a number of metabolic enzymes and carrier proteins by maintaining their proper quaternary structure in mitochondria (Table 1) (Schlame et al., 2000; Hatch, 1998). CL serves to anchor cytochrome $\mathrm{c}$ oxydase to the inner mitochondrial membrane. Two CL molecules were required to associate with cytochrome c oxydase to maintain its full enzyme activity. Complex III (cytochrome bc1) needs CL to maintain its quaternary structure (Gomez et al., 1999). Complex V was found to associate with CL molecules with high affinity (Eble et al., 1990).

Table 1. Genes involved in cardiolipin remodeling

\begin{tabular}{|c|c|c|c|}
\hline Name & Cell localization & functions & Related disorders \\
\hline tafazzin & $\begin{array}{l}\text { Cytoplasm and } \\
\text { mitochondria }(\mathrm{Xu} \text {, et } \\
\text { al., 2009) }\end{array}$ & $\begin{array}{l}\text { Transacylase function in } \\
\text { cardiolipin }(\mathrm{CL}) \\
\text { metabolism, } \\
\text { affect mitochondria } \\
\text { structure during its } \\
\text { differentiation(Rockman } \\
\text { et al., 2009) }\end{array}$ & $\begin{array}{l}\text { Barth syndrome, } \\
\text { Cardiomyopathy, dilated, 3A; } \\
\text { Endocardial fibroelastosis-2; } \\
\text { Left ventricular } \\
\text { noncompaction, X-linked; } \\
\text { Noncompaction of left } \\
\text { ventricular myocardium; male } \\
\text { sterility in Drosophila. }\end{array}$ \\
\hline MCLAT-1 & Mitochondria & $\begin{array}{l}\text { To acylate MLCL to CL } \\
\text { in the presence of }[1-14 C] \\
\text { linoleoyl coenzyme } A \text { in } \\
\text { vitro }\end{array}$ & $\begin{array}{l}\text { Affecting mitochondrial } \\
\text { Succinate dehydrogenase } \\
\text { activity }\end{array}$ \\
\hline ALCAT1 & $\begin{array}{l}\text { endoplasmic } \\
\text { reticulum }\end{array}$ & $\begin{array}{l}\text { transferred fatty acid from } \\
\text { fatty acyl coenzyme A to } \\
\text { MLCLto produce CL }\end{array}$ & $\begin{array}{l}\text { Improved glucose tolerance, } \\
\text { resistant to high fat diet } \\
\text { induced body weight gaining } \\
\text { and fat tissue store, insulin } \\
\text { sensitive. }\end{array}$ \\
\hline iPLA2 $\gamma$ & $\begin{array}{l}\text { mitochondria or } \\
\text { peroxisome } \\
\text { (Mancuso et al., } \\
2000 \text { ) }\end{array}$ & $\begin{array}{l}\text { Deacylation of CL at sn-2 } \\
\text { position (Mancuso et al., } \\
\text { 2000) }\end{array}$ & $\begin{array}{l}\text { decrease in ascorbate-induced } \\
\text { Complex IV-mediated oxygen } \\
\text { consumption; growth } \\
\text { retardation; }\end{array}$ \\
\hline CLD1 & $\begin{array}{l}\text { Mitochondria } \\
\text { (Beranek et al., } \\
\text { 2009) }\end{array}$ & $\begin{array}{l}\text { deacylation de novo } \\
\text { synthesized cardiolipin } \\
\text { (Beranek et al., 2009) }\end{array}$ & $\begin{array}{l}\text { Increased C16:0 decreased } \\
\text { C16:1 and C18:1 acyl chain in } \\
\text { CL (Beranek et al., 2009). }\end{array}$ \\
\hline
\end{tabular}


CL facilitates the protein complexes to form higher order complexes, which in turn improves the efficiency of ATP generation in oxidative phosphorylation. For example, it seems that CL is immobilized and cannot be exchanged with the exogenous radiolabeled CL. CL can only be released when the proteins are denatured, suggesting that CL binding is significant for the tertiary structure of the proteins with which it associates (Schlame et al., 2000; Schlame et al., 1991). To further understand the structure requirements of CL in protein interaction, many CL derivatives were tested. Negatively charged phosphate groups and the right number of acyl groups were important to its interaction with cytochrome oxidase (Powell et al., 1987). Many other CL derivatives such as CL dimethyl ester (no charge), monolysocardiolipin and acylcardiolipin also exhibited a stronger affinity for protein when compared to other class phospholipids (Schlame et al., 2000). It is also reported that the reduced cardiolipin level and other phospholipids in mitochondria inhibited the translation of electron transport chain proteins (Ostrander et al., 2001a).

Regulation of the release of cytochrome $\mathrm{c}$ from mitochondria by $\mathrm{CL}$ is a signal to trigger apoptosis (McMillin et al., 2002; Ostrander et al., 2001a). CL serves as a $\mathrm{Ca}^{2+}$ binding site, by which $\mathrm{Ca}^{2+}$ triggers mitochondrial membrane permeabilization (Orrenius et al., 2003). CL functions in preventing osmotic instability and uncoupling under the condition of higher respiration rates (Kriska et al., 2004; Girotti et al., 2004). Cl is also involved in other functions of mitochondria such as translocation of cholesterol from outer to the inner membrane, activation of mitochondrial cholesterol side-chain cleavage, and import of protein into mitochondria (Gasnier et al., 1998).

\section{CL Remodeling and the Related Enzymes}

\subsection{Remodeling}

De novo synthesized CL does not contain a high percentage of unsaturated fatty acyl groups. Therefore CL remodeling mechanisms were primarily predicted to achieve its special acyl content. The cardiolipin synthase (hCLS) did not display specific affinity for linoleoyl containing PG species compared to the oleoyl containing species (Houtkooper et al., 2006); the fatty acyl chains of CL exhibit an independent turnover non-relating to the remaining molecule (Landriscina et al., 1976); the molecular diacyl-species of PG, synthesized in isolated liver mitochondria were different from the diacyl-species of the phosphatidyl moieties in CL and the isocardiolipin identified in isolated mitochondria (Rüstow et al., 1989; Schlame et al., 1990); the molecular composition of CL was partially affected by the type of fatty acids provided in the diet (Shah et al., 2009). Currently, more attention is paid to CL remodeling because the abnormal CL profile and level were found in many diseases including Barth syndrome, diabetes, heart failure, and Parkinson disease (Sparagna et al., 2009; Su et al., 2005; Ramanadham et al., 2005; Widlansky et al., 2009; Bayir et al., 2009; Paradies et al., 2009).

Two possible models of cardiolipin remodeling have been reported. One model is Land Cycle-Acyl remodeling in mammalian phospholipid metabolism is through two steps: deacylation and reacylation that could occur in both microsome and mitochondria, the required enzymes include phospholipase to mediate deacylation and acyl tranferase to regulate reacylation (Figure 1) (Schlame, 2008; Eichberg, 1974). In this model, acyl group specific and position specific enzymes are needed. Though few genes were reported in the process, the detailed mechanism of CL remodeling is still elusive. The other model is tafazzin-controlled process-tafazzin controls the acyl group and position in the remodeling and maintains the cardiolipin content (Schlame, 2008). 


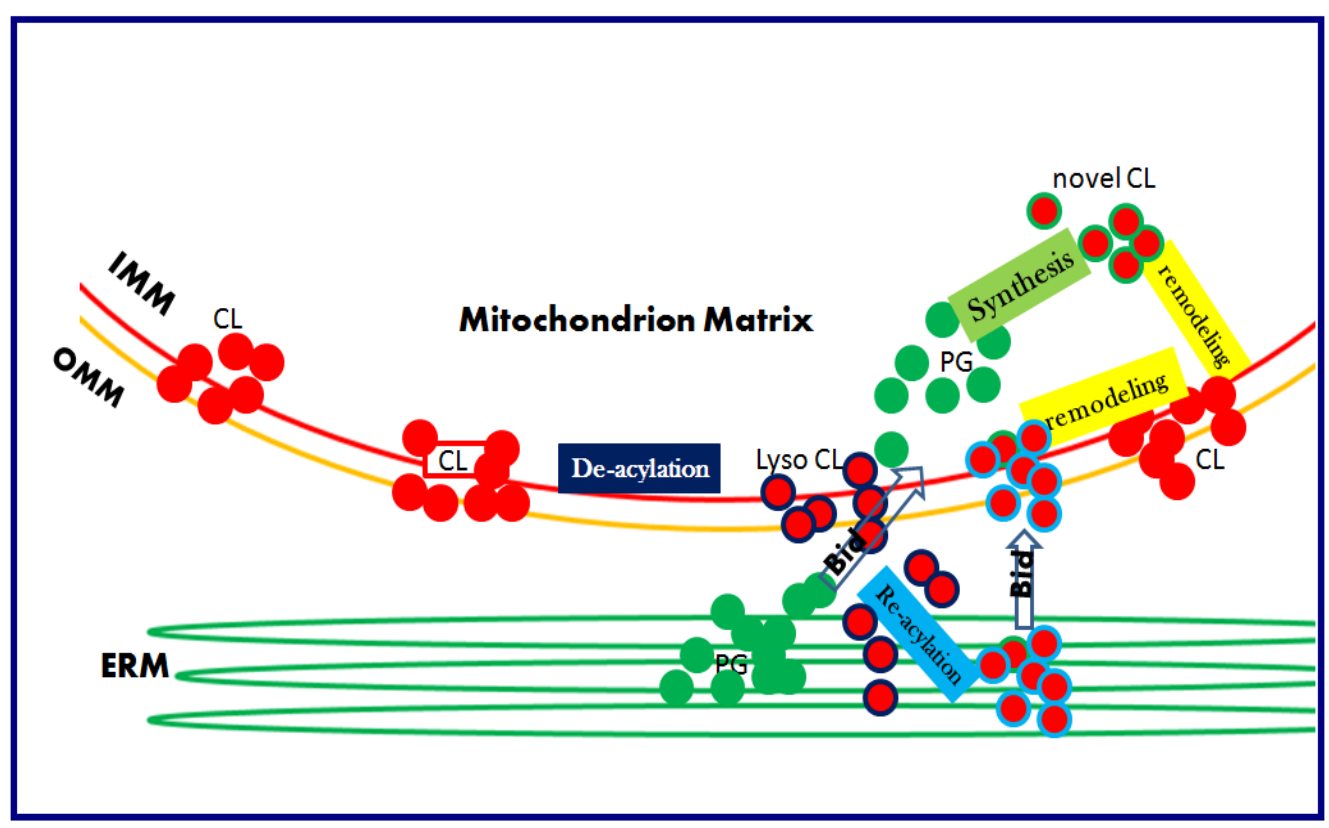

Figure 1. The schematic diagram showing the cardiolipin remodeling cycle

Cardiolipin (CL) is synthesized in mitochondrial matrix (labeled as green cycle with red filling) with precursors PG etc. CL is normally deacylated to mono- and di-lysocardiolipin (labeled as Lyso CL) that were transported to the ER for efficient reacylation by acyl-transferases. The recycled CL (labeled as light blue cycle with red filling), is then taken up, together with ER-produced precursors of CL, PG, by Bid (and other lipid transfer proteins), which move them to the mitochondria OM. Here, the CL equilibrates with the mitochondrial pool of $\mathrm{CL}$, constantly replenished by its local de novo biosynthesis, thereby closing the remodeling cycle.

\subsection{Enzymes in Cardiolipin Remodeling}

\subsubsection{Tafazzin Gene}

The TAZ gene mutation is responsible for Barth syndrome. The TAZ gene encodes a protein containing a domain homologous to phospholipid acyltransferases (Bione et al., 1996; Neuwald, 1997). The TAZ gene product is ubiquitously expressed and exists in several protein isoforms due to different splicing (Bione et al., 1996). Yeast complementation suggested one spliced variant of TAZ lacking exon 5 most likely represented the only physiological mRNA (Vaz et al., 2003; Xu et al., 2009). Patients with Barth syndrome exhibit abnormal CL content, such as decreased CL content, loss of dominant 18:2 fatty acyl content and loss of four linoleoyl CL (Schlame et al., 2002; Schlame et al., 2003). Barth syndrome underscores the significance of CL remodeling in vivo. Some studies on $\Delta t a z$ yeast mutant also suggested that defective CL remodeling with accumulation of MLCL decreased total CL content and increased saturated acyl chains in CL (Brandner et al., 2005; Ma et al., 2004). However, the enzyme activity of tafazzin in the cardiolipin remodeling was unknown. In vitro assay suggested that mutation of tafazzin showed decreased incorporation of linoleic acid into cardiolipin (Vreken et al., 2000). Other study showed that tafazzin catalyzes the substrate in such a way that acyl group from one phospholipid can be transferred to the other, no free acyl group is released if the acyl group receiver is absent and there is no intermediate enzyme-acyl formation step. Though tafazzin showed acyl group preference, it did not show phospholipid specificity; moreover, it did not show CoA or acyl-CoA dependent either (Schlame 2008; $\mathrm{Xu}$ et al., 2006). It was also reported that tafazzin could transfer the acyl group in the sn- 1 and sn-2 position of MLCL, thus it may function as an acyl position isomerase to transfer acyl groups' position in the cardiolipin molecule (Schlame, 2008)

\subsubsection{The Monolysocardiolipin Acyltransferase 1 (MLCLAT-1)}

The monolysocardiolipin acyltransferase 1, primarily purified from mitochondria of pig liver, is a protein with $74 \mathrm{kDa}$ molecular weight and 5.4 isoelectric point, exhibites in vitro monolysocardiolipin acyltransferase activity (Taylor et al., 2003). MLCLA -1 catalyzed linoleoyl-CoA and MLCL to form CL in a ping-pong reaction mechanism. MLCLAT-1 activity is $\mathrm{pH}$ and acyl-CoA dependant (its optimum $\mathrm{pH}$ is 7.0). MLCLAT-1 enzyme 
activity was 10 -fold greater with the unsaturated fatty-acyl CoA substrates than with palmitoyl-CoA. The expression of MLCLAT-1 protein was elevated in response to thyroxine treatment. The identified protein peptide matches to enoyl-coenzymeAhydratase, NAD-binding protein containing the Rossmann-fold for NAD(P) binding, and coenzyme A dehydrogenase (Taylor et al., 2009). Human MLCLAT-1(hMLCLAT-1) homologue was identified as a $59 \mathrm{kD}$ unknown protein and showed acyltransferase activity that could reacylate monolysocadiolipin on $\left[{ }^{14} \mathrm{C}\right]$ linoleoyl residues by an acyl-CoA dependent manner. The identified MLCLAT-1 showed a high activity (10 fold) for unsaturated acyl-CoAs over saturated palmitoyl-CoA, but no specificity for 18:2 substrates was demonstrated (Taylor et al., 2003; Taylor et al., 2009).

\subsubsection{Acyl-CoA: Lysocardiolipin Acyltransferase 1 (ALCAT1)}

ALCAT1, a $1.1 \mathrm{~kb}$ gene was cloned in 2004. ALCAT1 contains a highly conserved phosphate acyltransferase domain (Plsc), which is also found in the tafazzin gene, thus was predicted to be an acyl-CoA: lysocardiolipin acyltransferase (Neuwald, 1997; Cao et al., 2004). The mouse ALCAT1gene encodes a 376-amino acid protein with a predicted $44.4 \mathrm{kDa}$ molecular weight. ALCAT1 protein contains a conserved phosphate acyltransferase domain in the middle, several transmembrane sequences scattered at its $\mathrm{N}$-terminal and C-terminal and an endoplasmic reticulum (ER) localization sequence motif (KKXX) at the C-terminus. Multiple ALCAT1 orthologs were identified in many species except Caenorhabditis elegans and Saccharomyces cerevisiae. Northern analysis showed ALCAT1 highly expressed in heart and liver. ALCAT1 exhibits high substrate specificity for MLCL and DLCL in vitro. Recombinant ALCAT1 showed ER localization where the phospholipids and cardiolipin remodeling possible occurs (Cao et al., 2004). More information is needed to determine the relationship between MLCLAT-1 and ALCAT1 in vivo. It could be possible that ALCAT1 may function upstream of MLCLAT-1 in the remodeling process, but it needs more genetic evidence.

\subsubsection{Calcium-independent Phospholipase A2 (iPLA2)}

Phospholipases A2 (PLA2) is a diverse group of enzymes that hydrolyze the $s n-2$ fatty acids from phospholipids and play multiple roles in physiological functions. There are two classes of PLA2 - the secreted PLA2s and the cytosolic PLA2s. The secretory PLA2 (sPLA2) family has identified 10 isozymes, these enzymes are $\mathrm{Ca}^{2+}$ requiring, secretory enzymes with low molecular-weight which functioned in modification of eicosanoid generation, inflammation, host defense and atherosclerosis. The cytosolic PLA2 (cPLA2) family contains 3 kinds of enzymes: cPLA2alpha plays an essential role in the initiation of arachidonic acid (AA) metabolism, cPLA2alpha is tightly regulated by $\mathrm{Ca}^{2+}$ and phosphorylation; the $\mathrm{Ca}^{2+}$ - independent PLA2 (iPLA2) family contains 2 groups of enzymes (group VIA and VIB) which play a possible major role in phospholipid remodeling such as cardiolipin; the platelet-activating factor (PAF) acetylhydrolase (PAF-AH) family contains 4 enzymes representing a unique group which shows unusual substrate specificity toward PAF and/or oxidized phospholipids (Kudo et al., 2002).

Different from the cPLA2 family, the iPLA2 family contains a GXSTG consensus and possesses a consensus sequence for nucleotide binding; while cPLA2 family only contains a GXSGS consensus lipase motif (Mancuso et al., 2000). The human iPLA2 $\gamma$ was cloned as a homologue sequence to the iPLA2 $\alpha$ and iPLA2 $\beta$, which encodes a $88 \mathrm{kD}$ protein containing a lipase consensus sequence and mitochondrial and peroxisomal location signal sequences. The iPLA2 $\gamma$ was identified in many human tissues including heart, skeletal muscle, placenta, brain, liver, and pancreas. The enzyme demonstrated high lipase activity in sn-2-radiolabeled phosphatidylcholine (PC) and plasmenylcholine (PE) in Sf9 cells (Mancuso et al., 2000). Loss function of calcium-independent phospholipase $\mathrm{A}_{2} \gamma$ in mice showed several symptoms in hippocampal phospholipid change, cognitive dysfunction and bioenergetic dysfunction including abnormal CL content with elevated short acyl chain species, enlarged and degenerated mitochondria and reduced ascorbate-induced Complex IV-mediated oxygen consumption (Mancuso et al., 2009; Mancuso et al., 2007). In Drosophila, iPLA2-VIA, or calcium-independent phospholipase A2 group VIA suppressed the phenotype of male sterility in tafazzin mutant fly; and it also prevents cardiolipin depletion/monolysocardiolipin accumulation in this mutant; however, loss function of iPLA2-VIA did not affect the content of cardiolipin profile in wild type individuals. Moreover, the treatment of bromoenol lactone, the iPLA(2) inhibitor, could restore the homeostasis of cardiolipin the Barth syndrome patients' lymphoblasts (Malhotra et al., 2009).

\subsubsection{Cardiolipin-specific Deacylase 1(CLD1)}

Cld1p, the mitochondria cardiolipin-specific phospholipase, functioned upstream of Tazlp to generate monolyso-cardiolipin in yeast. Cld1p as a mitochondrial phospholipase (should be deacylase 1) deacylates de novo synthesized cardiolipin with strong substrate preference for palmitic acid residues to generate monolysocardiolipin for Tazlp-dependent reacylation in yeast (Beranek et al., 2009). Yet a mammalian 
homologue has not been identified so far.

\section{Disorders Caused by Abnormal Cardiolipin Remodeling}

\subsection{Barth Syndrome}

Human patients with Barth Syndrome were found to contain mutations in their tafazzin loci. Since loss of tafazzin function caused abnormality in cardiolipin level and species, dysfunction of mitochondria thus further affected the function of heart and skeleton muscle where mitochondria were highly demanded. In the study of patient tissue, mice and Drosophila model, identified CL defects included the reduced incorporation of linoleic acid into CL; reduced CL pool size; reduced tetra-linoleoyl cardiolipin, accumulated monolysocardiolipins and other cardiolipin species with aberrant acyl groups (Vreken et al., 2000; Acehan et al., 2011; Xu et al., 2006). The defects of cardiolipin profile identified in the patients indicated it is in a general way that tafazzin acts in the cardiolipin remodeling - to transfer the acyl chain of substrates without specificity in position and cardiolipin species. Affected mitochondrial structure included un-patterned cristae during mitochondrial differentiation, collapsed cristae with obliterated intracrista space (Vreken et al., 2000; Mancuso et al., 2000), which suggested that the organization of oxidative phosphorylation complexes on the inner membrane was impaired. Tafazzin gene in Homo sapiens is mainly expressed at high levels in cardiac and skeletal muscle, thus Barth Syndrome patients exhibited cardiomyopathy and skeletal muscle weakness (Acehan et al., 2011). The high sensitivity to the tetralinoleoyl cardiolipin species and content in these tissues caused the dysfunction of the organ.

\subsection{Parkinson Disease}

Parkinson disease is one type of central nervous system degenerative disease, the patients exhibited many symptoms including impaired functions of motor skills, impaired cognitive process and sleeping difficulties. Among them, the most prominent symptoms are the dysfunction of motor skills such as tremor, rigidity, retard movement and postural instability. It is known that mitochondria function was affected in Parkinson disease, such as dysfunction of mitochondrial complex I and reduced linkage of complex I/III activity to the electron transport chain (Schlame, 2008). Recently, this disease was found to link to the cardiolipin abnormality in brain due to oxidative stress, such as reduced cardiolipin content, acyl chain with reduced n-6 polyunsaturated FAs (fatty acids) and increased saturated FAs (Ellis et al., 2005). Cardiolipin formed complex with alpha-synuclein and cytochrome $\mathrm{C}$ to exert peroxidase activity to protect the neuron from damage by oxidative stress in the presence of $\mathrm{H} 2 \mathrm{O} 2$ (Bayir et al., 2009). There is no direct report if cardiolipin remodeling is impaired in the Parkinson disease but it is possible that cardiolipin remodeling dysfunction occurred in the affected neuron.

\subsection{Heart Failure}

Heart failure is the inability of heart to supply sufficient blood flow. The symptoms include orthopnea, cough, chronic venous congestion, ankle swelling and exercise intolerance. Cardiolipin abnormality such as reduced (18:2) (Schlame et al., 2000) cardiolipin due to decreased incorporation of 18:2 acyl chain to cardiolipin is found in the Spontaneously Hypertensive HF rat hearts cells, calcium-independent phospholipase A was found to be involved in heart failure since the incorporation was partially sensitive to the inhibition of bromoenol lactone (Zachman et al., 2010; Sparagna et al., 2007). Decreased MLCAT-1 activity and reduced tafazzin mRNA level were reported in the disorder of heart failure (Saini-Chohan et al., 2009).

\subsection{Metabolism Disorders}

Impaired energy generation by abnormality of cardiolipin in mitochondria is one of the causes of diabetes and obesity (Su et al., 2005; Widlansky et al., 2010). However, it is reported recently that the dysfunction of cardiolipin remodeling enzymes caused resistant to obesity and diabetes phenotypes under the high fat diet condition, which suggested a possible solution to deal with the metabolism disorders. Depletion of cardiolipin content and species in tissue culture have been known to be linked to mitochondria oxidative stress and aging response, which indicated the lost potential of mitochondrial inner membrane and may lead to oxidative phosphorylation uncoupling, depletion of ATP and activation of apoptotic cascade in the tissue (Sen et al., 2007). The loss function of mouse phospholipase iPLA2 gamma which was involved in the cardiolipin metabolism was reported to be resistant to high fat diet induced obesity and insulin resistance in vivo. The null mice of iPLA $\gamma$ exhibited cardiolipin abnormality including decreased levelof cardiolipin with an altered composition of molecular species in skeletal muscle; significantly decrease in State 3 respiration of skeleton muscle due to the uncoupling of phosphorylation and oxidative and increased fatty acid oxidation in adipose tissue under long term high fat diet treatment (Mancuso et al., 2010). Similarly, ALCAT1 null mice showed improved mitochondrial complex I activity, lipid oxidation, and insulin sensitivity and resistance to high fat diet induced obesity and 
insulin resistance (Yan, 2007; Yan et al., 2011; Li et al., 2010). These works suggested the depletion or reduction of cardiolipin could impair the energy generation and burn the metabolites substrates into futile.

\subsection{Recurrent Pregnancy Loss and Male Sterility}

The relationship between cardiolipin level and normal function of reproduction was primarily focused on the effect of autoimmune cardiolipin antibody on the female fertility. The anti-cardiolipin antibody (ACA) is one of factors responsible for recurrent pregnancy loss (RPL), about $15 \%$ of pregnant women have this condition. The patients are found to have elevated ACA serum levels and thrombosis, thrombocytopenia and RPL; moreover, defective embryonic implantation is a common link between unexplained infertility and recurrent miscarriage in the situation (Festin et al., 1997). The study of fly tafazzin mutant showed that cardiolipin level and species are important to the function of male sterility too. The tafazzin mutant in Drosophila demonstrated a male sterility phenotype. The tafazzin-deficient Drosophila model of Barth syndrome is featured with low cardiolipin concentration, abnormal cardiolipin fatty acyl composition, abnormal mitochondria structure, and poor motor function. The tafazzin deficiency in Drosophila disrupts the final stage of spermatogenesis, spermatid individualization, thus causes male sterility (Malhotra et al., 2009). Until now, there is no report on relationship between cardiolipin level and male sterility in human beings.

\section{Conclusions}

Due to the critical roles of CL in mitochondrial functions, lots of works have been done in the identification of related enzymes and their functions in CL remodeling. The remodeling process is regulated by different enzymes, suggesting the remodeling process involves multiple steps, although many questions need to be explored further such as how these enzymes reactions are regulated in vivo. Therefore, it can be anticipated that more detailed mechanisms of cardiolipin remodeling will be discovered and their implications in human diseases will be revealed.

\section{References}

Bayır, H., Kapralov, A. A., Jiang, J. F., Huang, Z. T., Tyurina, Y. Y., Zhao, Q., ... Kagan, V. E. (2009). Peroxidase mechanism of lipid-dependent cross-linking of synuclein with cytochrome $\mathrm{C}$ : protection against apoptosis versus delayed oxidative stress in Parkinson disease. J. Biol. Chem., 284(23), 15951-15969. http://dx.doi.org/10.1074/jbc.M900418200

Beranek, A., Rechberger, G., Knauer, H., Wolinski, H., Kohlwein, S. D., \& Leber, R. (2009). Identification of a cardiolipin-specific phospholipase encoded by the gene CLD1 (YGR110W) in yeast. J. Biol. Chem., 284(17), 11572-8. http://dx.doi.org/10.1074/jbc.M805511200

Bione, S., D'Adamo, P., Maestrini, E., Gedeon, A. K., Bolhuis, P. A., \& Toniolo, D. (1996). A novel X-linked gene, G4.5. is responsible for Barth syndrome. Nat Genet, 12(4), 385-9. http://dx.doi.org/10.1038/ng0496-385

Brandner, K., Mick, D. U., Frazier, A. E., Taylor, R. D., Meisinger, C., \& Rehling, P. (2005). Taz1, an outer mitochondrial membrane protein, affects stability and assembly of inner membrane protein complexes: implications for Barth Syndrome. Mol. Biol. Cell, 16(11), 5202-14. http://dx.doi.org/10.1091/mbc.E05-03-0256

Cao, J., Liu, Y., Lockwood, J., Burn, P., \& Shi, Y. (2004). A novel cardiolipin-remodeling pathway revealed by a gene encoding an endoplasmic reticulum-associated acyl-CoA:lysocardiolipin acyltransferase (ALCAT1) in mouse. J. Biol. Chem., 279(30), 31727-34. http://dx.doi.org/10.1074/jbc.M402930200

Devrim, A., Frederic, V., Riekelt, H. H., Jeanne, J., Vicky, M., Chonan, T., ... Zaza, K. (2011). Cardiac and skeletal muscle defects in a mouse model of human Barth syndrome. J. Biol. Chem., 286, 899-908. http://dx.doi.org/10.1074/jbc.M110.171439

Eble, K. S., Coleman, W. B., Hantgan, R. R., \& Cunningham, C. C. (1990). Tightly associated cardiolipin in the bovine heart mitochondrial ATP synthase as analyzed by $31 \mathrm{P}$ nuclear magnetic resonance spectroscopy. $J$. Biol. Chem., 265(32), 19434-40.

Eichberg, J. (1974). The reacylation of deacylated derivatives of diphosphatidylglycerol by microsomes and mitochondria from rat liver. J. Biol. Chem., 249(11), 3423-9.

Ellis, C. E., Murphy, E. J., Mitchell, D. C., Golovko, M. Y., Scaglia, F., Barceló-Coblijn, G. C. B., \& Nussbaum, R. L. (2005). Mitochondrial lipid abnormality and electron transport chain impairment in mice lacking $\begin{array}{lllll}\text { alpha-synuclein. } & \text { Mol. } & \text { Cell. } & \text { Biol., } & \text { 25(22), }\end{array}$ http://dx.doi.org/10.1128/MCB.25.22.10190-10201.2005 
Festin, M. R., Limson, G. M., \& Maruo, T. (1997). Autoimmune causes of recurrent pregnancy loss. Kobe J. Med. Sci., 43(5), 143-57.

Gasnier, F., Rey, C., Hellio, Le Graverand, M. P., Benahmed, M., \& Louisot, P. (1998). Hormone-induced changes in cardiolipin from Leydig cells: possible involvement in intramitochondrial cholesterol translocation. Biochem. Mol. Biol. Int., 45(1), 93-100.

Girotti, A. W., \& Kriska, T. (2004). Role of lipid hydroperoxides in photo-oxidative stress signaling. Antioxid Redox Signal, 6(2), 301-10. http://dx.doi.org/10.1089/152308604322899369

Gomez, B. J., \& Robinson, N. C. (1999). Phospholipase digestion of bound cardiolipin reversibly inactivates bovine cytochrome bc. Biochemistry, 38(28), 9031-8. http://dx.doi.org/10.1021/bi990603r

Haines, T. H., \& Dencher, N. A. (2002). Cardiolipin: a proton trap for oxidative phosphorylation. FEBS Lett, 528(1-3), 35-9. http://dx.doi.org/10.1016/S0014-5793(02)03292-1

Hatch, G. M. (1998). Cardiolipin: biosynthesis, remodeling and trafficking in the heart and mammalian cells (Review). Int. J. Mol Med, 1(1), 33-41.

Hoch, F. L. (1992). Cardiolipins and biomembrane function. Biochim. Biophys. Acta, 1113, 71-133.

Houtkooper, R. H., Akbari, H., van Lenthe, H., Kulik, W., Wanders, R. J., Frentzen, M., \& Vaz, F. M. (2006). Identification and characterization of human cardiolipin synthase. FEBS Lett, 580(13), 3059-64. http://dx.doi.org/10.1016/j.febslet.2006.04.054

Jiang, F., Ryan, M. T., Schlame, M., Zhao, M., Gu, Z., Klingenberg, M., ... Greenberg, M. L. (2000). Absence of cardiolipin in the crd1 null mutant results in decreased mitochondrial membrane potential and reduced mitochondrial function. J. Biol Chem, 275(29), 22387-94. http://dx.doi.org/10.1074/jbc.M909868199

Keenan, W., Awasthi, Y. C., \& Crane, F. L. (1970). Cardiolipin from beef heart mitochondria: Fatty acid positioning and molecular species distribution. Biochem. Biophys. Res. Comm, 40, 1102-9. http://dx.doi.org/10.1016/0006-291X(70)90908-3

Kriska, T., \& Girotti, A. W. (2004). Separation and quantitation of peroxidized phospholipids using high-performance thin-layer chromatography with tetramethyl-p-phenylenediamine detection. Anal Biochem, 327(1), 97-106. http://dx.doi.org/10.1016/j.ab.2003.12.021

Kudo, I., \& Murakami, M. (2002). Phospholipase A2 enzymes. Prostaglandins Other Lipid Mediat, 68-69, 3-58. http://dx.doi.org/10.1016/S0090-6980(02)00020-5

Landriscina, C., Megli, F. M., \& Quagliariello, E. (1976). A general method for phospholipid polar head spin labeling. Synthesis of diphosphatidylglycerol-2(2' 2', 5', 5',-tetramethylpyrroline-N-oxide) carboxylate. Anal Biochem, 76(1), 292-9. http://dx.doi.org/10.1016/0003-2697(76)90287-6

LeCocq, J., \& Ballou, C. E. (1964). On The Structure of Cardiolipin. Biochemistry, 3, 976-80. http://dx.doi.org/10.1021/bi00895a023

Li, J., Romestaing, C., Han, X. (2010). Cardiolipin remodeling by ALCAT1 links oxidative stress and mitochondrial dysfunction to obesity. Cell Metab, 12(2), 154-165. http://dx.doi.org/10.1016/j.cmet.2010.07.003

Ma, L., Vaz, F. M., Gu, Z., Wanders, R. J., \& Greenberg, M. L. (2004). The human TAZ gene complements mitochondrial dysfunction in the yeast taz1Delta mutant. Implications for Barth syndrome. J. Biol. Chem., 279(43), 44394-9. http://dx.doi.org/10.1074/jbc.M405479200

Maeda, A., Zhu, J., \& Na, H. M. (2009) .Peroxidase mechanism of lipid-dependent cross-linking of synuclein with cytochrome C: protection against apoptosis versus delayed oxidative stress in Parkinson disease. $J$. Biol. Chem., 284(23), 15951-69. http://dx.doi.org/10.1074/jbc.M900418200

Malhotra, A., Edelman-Novemsky, I., Xu, Y., Plesken, H., Ma, J., Schlame, M., \& Ren, M. (2009). Role of calcium-independent phospholipase A2 in the pathogenesis of Barth syndrome. Pro.c Natl. Acad. Sci. USA, 106(7), 2337-41. http://dx.doi.org/10.1073/pnas.0811224106

Mancuso, D. J., Jenkins, C. M., \& Gross, R. W. (2000). The genomic organization, complete mRNA sequence, cloning, and expression of a novel human intracellular membrane-associated calcium-independent phospholipase A(2). J. Biol. Chem., 275, 9937-45. http://dx.doi.org/10.1074/jbc.275.14.9937

Mancuso, D. J., Kotzbauer, P., \& Wozniak, D. F. (2009). Genetic ablation of calcium-independent phospholipase A2 \{gamma\} leads to alterations in hippocampal cardiolipin content and molecular species distribution, 
mitochondrial degeneration, autophagy, and cognitive dysfunction. J. Biol. Chem., 284(51), 35632-44. http://dx.doi.org/10.1074/jbc.M109.055194

Mancuso, D. J., Sims, H. F., Han, X., Jenkins, C. M., Guan, S. P., Yang, K., ... Gross, R. W. (2007). Genetic ablation of calcium-independent phospholipase A2gamma leads to alterations in mitochondrial lipid metabolism and function resulting in a deficient mitochondrial bioenergetic phenotype. J. Biol Chem, 282(48), 34611-22. http://dx.doi.org/10.1074/jbc.M707795200

Mancuso, D. J., Sims, H. F., Yang, K., Kiebish, M. A., Su, X., Jenkins, C. M., ... Gross, R. W. (2010). Genetic Ablation of Calcium-independent Phospholipase A2 $\gamma$ Prevents Obesity and Insulin Resistance During High Fat Feeding by Mitochondrial Uncoupling and Increased Adipocyte Fatty Acid Oxidation. J. Biol. Chem., 285, 36495-510. http://dx.doi.org/10.1074/jbc.M110.115766

McMillin, J. B., \& Dowhan, W. (2002). Cardiolipin and apoptosis. Biochim Biophys Acta, 1585(2-3), 97-107.

Neuwald, A. F. (1997). An unexpected structural relationship between integral membrane phosphatases and soluble haloperoxidases. Protein Sci, 6(8), 1764-7. http://dx.doi.org/10.1002/pro.5560060817

Neuwald, A. F. (1997). Barth syndrome may be due to an acyltransferase deficiency. Curr Biol, 7(8), 465-6. http://dx.doi.org/10.1016/S0960-9822(06)00237-5

Orrenius, S., Zhivotovsky, B., \& Nicotera, P. (2003). Regulation of cell death: the calcium-apoptosis link. Nat Rev Mol Cell Biol, 4(7), 552-65. http://dx.doi.org/10.1038/nrm1150

Ortiz, A., Killian, J. A., Verkleij, A. J., \& Wilschut, J. (1999). Membrane fusion and the lamellar-to-inverted-hexagonal phase transition in cardiolipin vesicle systems induced by divalent cations. Biophys J., 77(4), 2003-14. http://dx.doi.org/10.1016/S0006-3495(99)77041-4

Ostrander, D. B., Sparagna, G. C., Amoscato, A. A., McMillin, J. B., \& Dowhan, W. (2001). Decreased cardiolipin synthesis corresponds with cytochrome c release in palmitate-induced cardiomyocyte apoptosis. J. Biol Chem, 276(41), 38061-7. http://dx.doi.org/10.1074/jbc.M103689200

Ostrander, D. B., Zhang, M., Mileykovskaya, E., Rho, M., \& Dowhan, W. (2001). Lack of mitochondrial anionic phospholipids causes an inhibition of translation of protein components of the electron transport chain. A yeast genetic model system for the study of anionic phospholipid function in mitochondria. J. Biol. Chem., 276(27), 25262-72.

Pangborn, M. (1942). Isolation and purification of a serologically active phospholipid from beef heart. J. Biol. Chem., 143, 247-56.

Paradies, G., Petrosillo, G., Paradies, V., \& Ruggiero, F. M. (2009). Role of cardiolipin peroxidation and $\mathrm{Ca}^{2+}$ in mitochondrial dysfunction and disease. Cell Calcium, 45(6), 643-50. http://dx.doi.org/10.1016/j.ceca.2009.03.012

Powell, G. L., \& Jacobus, J. (1974). The nonequivalence of the phosphorus atoms in cardiolipin. Biochemistry, 13, 4024-6. http://dx.doi.org/10.1021/bi00716a032

Powell, G. L., Knowles, P. F., \& Marsh, D. (1987). Spin-label studies on the specificity of interaction of cardiolipin with beef heart cytochrome oxidase. Biochemistry, 26(25), 8138-45. http://dx.doi.org/10.1021/bi00399a018

Ramanadham, S., Hsu, F. F., Zhang, S., Jin, C., Bohrer, A., Song, H. W., \& ... Turk, J. (2004). Apoptosis of insulin-secreting cells induced by endoplasmic reticulum stress is amplified by overexpression of group VIA calcium-independent phospholipase A2 (iPLA2 beta) and suppressed by inhibition of iPLA2 beta. Biochemistry, 43(4), 918-30. http://dx.doi.org/10.1021/bi035536m

Rockman, H. A., Stokes, D. L., \& Schlame, M. (2009). Distinct effects of tafazzin deletion in differentiated and undifferentiated mitochondria. Mitochondrion, 9(2), 86-95. http://dx.doi.org/10.1016/j.mito.2008.12.001

Rüstow, B., Schlame, M., Rabe, H., Reichmann, G., \& Kunze, D. (1989). Species pattern of phosphatidic acid, diacylglycerol, CDP-diacylglycerol and phosphatidylglycerol synthesized de novo in rat liver mitochondria. Biochim Biophys Acta, 1002(2), 261-3.

Saini-Chohan, H. K., Holmes, M. G., Chicco, A. J., Taylor, W. A., Moore, R. L., McCune, S. A., ... Sparagna, G. C. (2009). Cardiolipin biosynthesis and remodeling enzymes are altered during development of heart failure. J. Lipid Res., 50(8), 1600-8. http://dx.doi.org/10.1194/jlr.M800561-JLR200 
Schlame, M., \& Haldar, D. (1993). Cardiolipin is synthesized on the matrix side of the inner membrane in rat liver mitochondria. J. Biol. Chem., 268, 74-9.

Schlame, M., \& Rüstow, B. (1990). Lysocardiolipin formation and reacylation in isolated rat liver mitochondria. Biochem J., 272(3), 589-95.

Schlame, M. (2008). Cardiolipin synthesis for the assembly of bacterial and mitochondrial membranes. J. Lipid Res., 49(8), 1607-20. http://dx.doi.org/10.1194/jlr.R700018-JLR200

Schlame, M., Beyer, K., Hayer-Hartl, M., \& Klingenberg, M. (1991). Molecular species of cardiolipin in relation to other mitochondrial phospholipids. Is there an acyl specificity of the interaction between cardiolipin and the ADP/ATP carrier? Eur J. Biochem, 199(2), 459-66. http://dx.doi.org/10.1111/j.1432-1033.1991.tb16144.x

Schlame, M., Kelley, R. I., Feigenbaum, A. (2003). Phospholipid abnormalities in children with Barth syndrome. J. Am Coll Cardiol, 42(11), 1994-9. http://dx.doi.org/10.1016/j.jacc.2003.06.015

Schlame, M., Rua, D., \& Greenberg, M. L. (2000). The biosynthesis and functional role of cardiolipin. Prog Lipid Res, 39, 257-88. http://dx.doi.org/10.1016/S0163-7827(00)00005-9

Schlame, M., Towbin, J. A., Heerdt, P. M., Jehle, R., DiMauro, S. \& Blanck, T. J. (2002). Deficiency of tetralinoleoyl-cardiolipin in Barth syndrome. Ann. Neurol, 51(5), 634-7. http://dx.doi.org/10.1002/ana.10176

Sen, T., Sen, N., Jana, S., Khan, F. H., Chatterjee, U., \& Chakrabarti, S. (2007). Depolarization and cardiolipin depletion in aged rat brain mitochondria: relationship with oxidative stress and electron transport chain activity. Neurochem Int., 50(5), 719-25. http://dx.doi.org/10.1016/j.neuint.2007.01.007

Shah, K. B., Duda, M. K., O'Shea, K. M., Sparagna, G. C., Chess, D. J., Khairallah, R. J., ... Stanley, W. C. (2009). The cardioprotective effects of fish oil during pressure overload are blocked by high fat intake: role of cardiac phospholipid remodeling. Hypertension, 54(3), 605-11. http://dx.doi.org/10.1161/HYPERTENSIONAHA.109.135806

Sparagna, G. C., \& Lesnefsky, E. J. (2009). Cardiolipin remodeling in the heart. J. Cardiovasc Pharmacol, 53(4), 290-301. http://dx.doi.org/10.1097/FJC.0b013e31819b5461

Sparagna, G. C., Chicco, A. J., Murphy, R. C., Schlame, M., Kelley, R. I., Feigenbaum, A., ... Blanck, T. J. J. (2007). Loss of cardiac tetralinoleoyl cardiolipin in human and experimental heart failure. J. Lipid Res, 48(7), 1559-70. http://dx.doi.org/10.1194/jlr.M600551-JLR200

Su, X., Han, X., Mancuso, D. J., Abendschein, D. R., \& Gross, R. W. (2005). Accumulation of long-chain acylcarnitine and 3-hydroxy acylcarnitine molecular species in diabetic myocardium: identification of alterations in mitochondrial fatty acid processing in diabetic myocardium by shotgun lipidomics. Biochemistry, 44(13), 5234-45. http://dx.doi.org/10.1021/bi047773a

Taylor, W. A., \& Hatch, G. M. (2003). Purification and characterization of monolysocardiolipin acyltransferase from pig liver mitochondria. J. Biol. Chem., 278(15), 12716-21. http://dx.doi.org/10.1074/jbc.M210329200

Taylor, W. A., \& Hatch, G. M. (2009). Identification of the human mitochondrial linoleoyl-coenzyme A monolysocardiolipin acyltransferase (MLCL AT-1). J. Biol. Chem., 284(44), 30360-71. http://dx.doi.org/10.1074/jbc.M109.048322

Tuominen, E. K., Wallace, C. J., \& Kinnunen, P. K. (2002). Phospholipid-cytochrome c interaction: evidence for the extended lipid anchorage. J. Biol. Chem., 277(11), 8822-6. http://dx.doi.org/10.1074/jbc.M200056200

Vaz, F. M., Houtkooper, R. H., Valianpour, F., Barth, P. G., \& Wanders, R. J. (2003). Only one splice variant of the human TAZ gene encodes a functional protein with a role in cardiolipin metabolism. J. Biol. Chem., 278(44), 43089-94. http://dx.doi.org/10.1074/jbc.M305956200

Vreken, P., Valianpour, F., Nijtmans, L. G., Grivell, L. A., Plecko, B., Wanders, R. J., \& Barth, P. G. (2000). Defective remodeling of cardiolipin and phosphatidylglycerol in Barth syndrome. Biochem Biophys Res Commun, 279(2), 378-82. http://dx.doi.org/10.1006/bbrc.2000.3952

Widlansky, M. E., Wang, J., Shenouda, S. M., Jin, C., Bohrer, A., Song, F. W., ... Turk, J. (2004). Apoptosis of insulin-secreting cells induced by endoplasmic reticulum stress is amplified by overexpression of group VIA calcium-independent phospholipase A2 (iPLA2 beta) and suppressed by inhibition of iPLA2 beta. Biochemistry, 43(4), 918-30. http://dx.doi.org/10.1021/bi035536m 
Widlansky, M. E., Wang, J., Shenouda, S. M., Hagen, T. M., Smith, A. R., Kizhakekuttu, T. J., ... Vita, J. A. (2010). Altered mitochondrial membrane potential, mass, and morphology in the mononuclear cells of humans with type 2 diabetes. Transl. Res., 156(1), 15-25. http://dx.doi.org/10.1016/j.trsl.2010.04.001

Xu, Y., Condell, M., Plesken, H., Edelman-Novemsky, I., Ma, J., Ren, M., \& Schlame, M. (2006). A Drosophila model of Barth syndrome. Proc. Natl. Acad. Sci. USA, 103(31), $11584-8$. http://dx.doi.org/10.1073/pnas.0603242103

Xu, Y., Malhotra, A., Ren, M., \& Schlame, M. (2006). The enzymatic function of tafazzin. J. Biol. Chem., 281, 39217-24. http://dx.doi.org/10.1074/jbc.M606100200

Xu, Y., Zhang, S., Malhotra, A., Edelman-Novemsky, I., Ma, J. P., Kruppa, A., ... Schlame, M. (2009) Characterization of tafazzin splice variants from humans and fruit flies. J. Biol. Chem., 284(42), $29230-9$. http://dx.doi.org/10.1074/jbc.M109.016642

Yan, Y., \& Kang, B. (2010). Resistance of Alcat1 Null Mice to High Fat Diet Induced Obesity and Impaired Glucose Tolerance. Int. J. Biol, 3(2), 11-22.

Yan, Y., (2007). The function of lysocardiolipin acyl transferase(ALCAT1) in controlling mice energy homeostasis. Penn State University thesis.

Zachman, D. K., Chicco, A. J., McCune, S. A., Murphy, R. C., Moore, R. L., \& Sparagna, G. C. (2010). The role of calcium-independent phospholipase A2 in cardiolipin remodeling in the spontaneously hypertensive heart failure rat heart. J. Lipid Res., 51(3), 525-34. http://dx.doi.org/10.1194/jlr.M000646 